\title{
Le deuil du père en pays goin (Burkina Faso)
}

Mourning the Father among the Guin (Burkina Faso)

\section{Michèle Dacher}

\section{OpenEdition}

Journals

Édition électronique

URL : http://journals.openedition.org/span/1131

DOI : 10.4000/span. 1131

ISSN : 2268-1558

\section{Éditeur}

École pratique des hautes études. Sciences humaines

\section{Édition imprimée}

Date de publication : 1 novembre 1989

Pagination : 75-104

ISSN : 0294-7080

\section{Référence électronique}

Michèle Dacher, «Le deuil du père en pays goin (Burkina Faso) », Systèmes de pensée en Afrique noire [En ligne], 9 | 1989, mis en ligne le 15 octobre 2013, consulté le 02 mai 2019. URL : http:// journals.openedition.org/span/1131; DOI : 10.4000/span.1131 


\section{LE DEUIL DU PERE EN PAYS GOIN (BURKINA FASO)}

par

\section{Michèle Dacher}

Comme dans de nombreuses sociétés "voltaïques", les cérémonies funéraires goin (sud-ouest du Burkina Faso) on lieu en trois temps: l'enterrement, une période intermédiaire, et les funérailles. Ces dernières comportent plusieurs séquences, la première étant le rite de l'ambalma. A l'issue de ce rite, le mort a été éloigné des vivants et rapproché des ancêtres. Pour les vivants, l'ambalma met fín aux prohibitions qui pesaient sur les deuilleurs, ce qui permet notamment, au lendemain même du rite, le remariage des veuves avec les héritiers du défunt. Mais le double mouvement qu'a enclenché l'ambalma (mort rapproché des ancêtres et vivants libérés des comportements de deuil) ne s'achève pas avec la levée de deuil. D'autres rites seront nécessaires pour que le processus d'ancestralisation soit mené à son terme. La séquence finale des funérailles consiste en l'insertion d'une pierre supplémentaire dans l'autel des ancêtres (rangées de pierres fixées dans le haut du mur extérieur de la maison, près de la porte d'entrée). Le rite marque l'accès du défunt au statut d'ancêtre et donc, son intégration au groupe des ancêtres auxquels un culte est rendu.

Systèmes de pensée en Afrique Noire, 9, 1989 
Dans cette société matrilinéaire, ou à prédominance matrilinéaire $^{1}$, les parents soumis au deuil sont, pour un défunt masculin, ses enfants et ses épouses - c'est-à-dire des parents hors filiationtandis que ses maternels n'y sont pas astreints. Pour une défunte, les comportements de deuil concernent ses enfants et son mari, mais ils sont davantage laissés au libre choix des individus qu'imposés par la coutume.

Dans ce travail, nous nous proposons de décrire le rite ambalma et les comportements de deuil observés depuis le décès jusqu'à la fin du rite. Nous chercherons ensuite à saisir la logique qui soustend le partage des parents du mort en "parents en deuil" et "parents hors deuil". Nous nous attacherons tout d'abord aux comportements de deuil et au rite de levée de deuil tels qu'ils sont observés pour un défunt de sexe masculin; nous indiquerons ensuite les variantes lorsque deuil et ambalma sont accomplis pour une femme. Dans la seconde partie de l'article, nous exposerons rapidement l'organisation familiale. Nous verrons que le deuil ne concerne pas le groupe de filiation (matrilinéaire) mais les alliés et les descendants et que, pour un défunt masculin, le rite est effectué conjointement par son père et par son fils. Nous analyserons le concept de paternité dans cette société matrilinéaire et nous montrerons que la part biologique de la paternité est totalement déniée au profit d'une définition symbolique. La paternité, qui est corrélative de l'alliance, ne devient effective qu'après l'accomplissement $\mathrm{du}$ rite de mariage; elle est également fondée, de manière beaucoup moins visible mais tout aussi fondamentale, par le rite ambalma. Nous constaterons d'ailleurs qu'il

1 Les Goin (ou Gouin ou Ciranba) comptent environ 50000 personnes au Burkina Faso et quelques milliers en côte-d'Ivoire. Cette société lignagère est traditionnellement acéphale, caractère qui demeure en partie malgré le renforcement des unités villageoises et la création de chefs de village par l'administration coloniale. L'organisation familiale était matrilinéaire; actuellement on peut la qualifier de bilinéaire à prédoninance matrilinéaire, tendant à devenir patrilinéaire dans les villes. Le système de parenté est de type crow. Les Goin sont des agriculteurs passionnés et relativement favorisés par la nature (isohyète 1300). Cependant les migrations en Côte-d'Ivoire, saisonnières ou de plus longue durée, sont anciennes et importantes. La langue goin (ou cirma), qui n'a encore jamais été analysée, appartient au groupe des langues voltaïques. 
existe une équivalence structurelle et formelle entre les rites matrimoniaux et les rites funéraires.

I - COMPORTEMENTS DE DEUIL ET RITE DE LEVEE DE DEUIL

Le terme ambalma vient du mot mbala ou mabala qui signifie mauvais. Faire l'ambalma, c'est enlever la mauvaise chose du mort ${ }^{2}$. Pour certains informateurs, il s'agit de purifier le mort du mal qu'il a commis pendant sa vie et qui l'empêche d'être accueilli par ses ancêtres; pour d'autres, le rite sert à libérer les épouses et les enfants des dangers que le défunt leur fait courir. Nous verrons que ces deux points de vue ne se contredisent pas mais se complètent.

L'ambalma doit être accompli pour tous les adultes mariés, avec ou sans enfants. En ce qui concerne les enfants, l'ambalma n'est pratiqué que pour le premier-né de chaque épouse, y compris s'il est mort-né. Le rite prend alors un sens différent : il s'agit d'éviter la répétition de ce malheur en dissuadant l'esprit ancestral, "incarné" dans un nouvel être, de continuer ses allées et venues entre le monde des vivants et celui des morts. Les cérémonies funéraires des enfants se limitent à un ambalma extrêmement écourté, exécuté par les parents au moment de l'enterrement.

Pour un homme, l'ambalma est accompli par un ou plusieurs de ses fils (réels ou classificatoires) et par ses épouses. La terminologie de parenté étant de type crow, entrent dans la catégorie des fils, pour un Ego masculin, les fils de tous les membres masculins de son matrilignage (voir schéma, page suivante). Les fils classificatoires d'Ego sont également ceux de tous les membres masculins de son lignage. Ils peuvent donc accomplir l'ambalma pour n'importe lequel de leurs pères classificatoires.

2 On dit parfois faire le maama, c'est-à-dire la chose, l'affaire. 


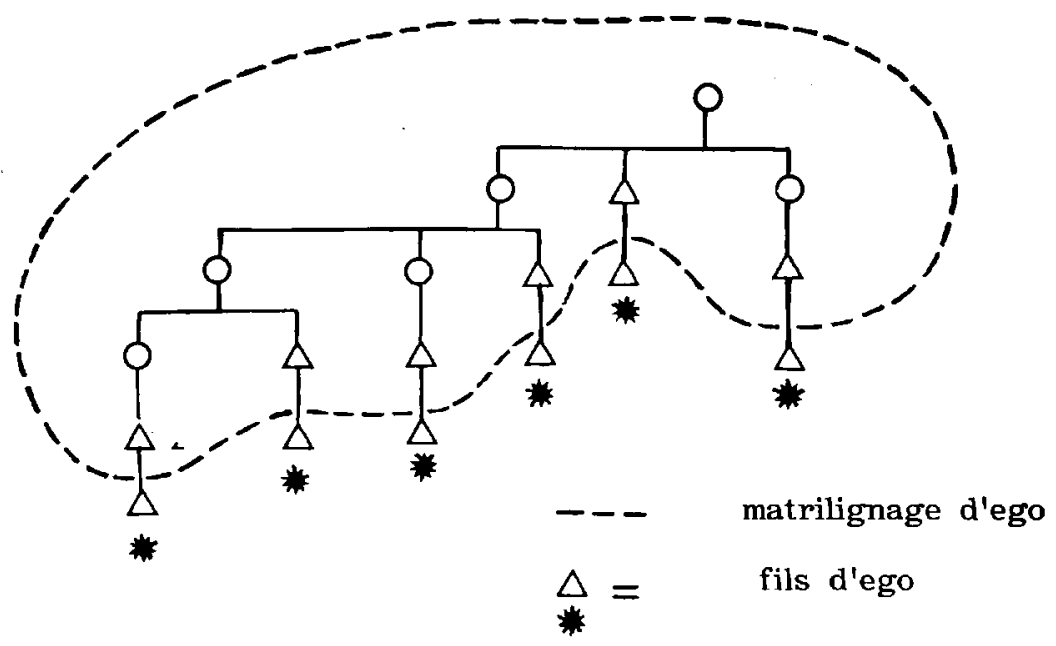

Le rite est généralement accompli par plusieurs fils dont l'un tient le rôle principal. Les frères utérins âgés, réels ou classificatoires, se réunissent en conseil de famille pour se répartir les fils chargés de leur ambalma respectif. Garder son fils réel pour son propre rituel risque d'être interprété comme un signe de mésentente dans la famille ou comme un excès d'individualisme.

Ces échanges de fils entre frères utérins pallient les aléas démographiques qui pourraient priver un homme de descendance mâle. Ils illustrent le fait que le matrilignage est, vis-à-vis de l'extérieur, un "groupe en corps" (corporate group) dont les membres sont substituables les uns aux autres; ils affirment la prééminence des liens utérins sur tout autre lien de parenté. Ceci ne signifie pas que le matrilignage dénie à la relation agnatique sa spécificité ou son importance - la description des rites de deuil le montre - mais qu'il veille à ce que cette relation demeure une relation entre deux individus, un père et un fils, et ne devienne pas un principe susceptible de légitimer l'instauration de lignées agnatiques dont l'autonomie et les intérêts concurrenceraient ceux des maternels.

Pour une femme, l'ambalma est accompli par une fille de soeur, à défaut par une fille directe. 
On ne fait l'ambalma qu'une seule fois dans sa vie pour un parent de même sexe : le fils pour son père, la fille pour sa mère. En tant qu'épouse, une femme participera à l'ambalma effectué pour son premier mari (mais non pour les éventuels autres conjoints, ces derniers n'étant, au regard de l'institution léviratique, que des substituts du défunt ${ }^{3}$ ). En tant qu'époux, un homme accomplit l'ambalma de sa première épouse s'il l'a obtenue par mariage direct et non par héritage (il n'a pas à faire l'ambalma des veuves dont il a hérité puisque son remariage avec elles ne constitue pas une nouvelle alliance mais la reconduction du lien conjugal conclu avec l'ensemble du matrilignage). Pour les épouses suivantes, l'exécution de l'ambalma est laissée au libre choix du mari.

La période de deuil s'étend entre le décès et l'ambalma, qui marque théoriquement le début des funérailles. La coutume fixe la durée de cette période à trois semaines de cing jours pour un homme et quatre semaines pour une femme, soit respectivement quinze et vingt jours. En pratique, les difficultés matérielles obligent souvent les familles, d'une part à allonger la période intermédiaire, d'autre part à détacher le rite de sortie de deuil du reste des funérailles : celles-ci pourront être repoussées à la prochaine saison sèche, au pire à l'année suivante. Mais le retard apporté à la célébration de l'ambalma ne saurait excéder quelques mois sans mettre gravement en danger la famille du défunt.

\section{De l'enterrement à l'ambalma}

L'acteur principal de l'ambalma, fils réel ou classificatoire du mort, se nomme ambalmatiyo (celui de l'ambalma). Son ou ses frères, éventuellement responsables avec lui du rituel, de même que les veuves concernées par la levée de deuil, sont également appelés ambalmatiyo (pl. ambalmatama). Dans la pratique, le terme a tendance

3 Même en cas de remariage légal avec un étranger au lignage de son premier mari, une veuve ne fera pas de second ambalma. 
à s'étendre à tous les enfants et à toutes les veuves, ainsi qu'aux parents impliqués économiquement ou rituellement dans la cérémonie.

Dans le village de Gouera (mais pas dans la totalité du pays goin), l'ambalmatiyo doit faire la toilette mortuaire de son père. On peut alors le désigner par le terme de tiasartiyo, "celui qui verse l'eau sur le sexe"4. Les gestes de la toilette mortuaire, comme la plupart de ceux qui ont trait à la mort, sont exécutés "à l'envers", comme s'ils étaient vus dans un miroir. Ainsi, l'ambalmatiyo pénètre dans la case mortuaire, la nuit, à reculons, la tête enfoncée dans un panier. Guidé par des vieillards qui lui ont mis des rameaux feuillus entre les mains, il va à reculons jusqu'à la dépouille paternelle sur laquelle il esquisse quelques gestes symboliques de toilette. Le corps sera ensuite complètement lavé à l'eau et au savon par les vieillards, frotté au beurre de karité, parfumé, vêtu d'un cache-sexe de coton blanc retenu par une lanière de cuir, puis enveloppé d'un linceul blanc ${ }^{5}$.

A l'enterrement, la fille aînée du mort conduit le cortège de la maison à la tombe, vêtue du boubou de son père; ses frères la suivent ${ }^{6}$. Au moment de mettre le corps en terre les veuves dissimulent le spectacle aux jeunes enfants: elles leur mettent la tête dans un panier et les tirent à reculons vers l'intérieur de la concession tout en faisant retentir des lamentations.

Généralement les épouses infidèles doivent fuir la concession dès le décès de leur mari. Elles ne reparaîtront, couvertes de bouse

4 A Banfora, l'ambalmatizo n'est pas tenu à une toilette mortuaire, réelle ou symbolique. Il doit seulement avoir vu le cadavre de son père.

5 Les acteurs de la toilette peuvent être également de vieilles parentes maternelles ou encore les dorama du mort, c'est-à-dire les fermes pour lesquelles il a joué un rôle d'intermédiaire et de témoin matrimonial. Si celles-ci sont encore jeunes, elles payent le savon et délèguent l'exécution de la tâche à des fermes âgées. La participation symbolique du tiasartiyo à la toilette mortuaire peut également être marquée de la manière suivante : la tête enfoncée dans un panier, il tient le bras gauche de la ferme qui lave le corps, car il ne doit ni voir ni toucher lui-même le cadavre.

6 In mythe raconte l'origine de la prééminence des filles sur les fils : un père, voulant mettre ses enfants à l'épreuve, les enmena en brousse. Soudain, il s'éloigna et leur dít de l'attendre. Il "mit sa voix dans un petit panier" et imita les rugissements du lion. Ses fils se sauvèrent affolés. Ses filles se précipitèrent à son secours. Alors il dit : "Dorénavant, à mon enterrement, les filles passeront devant les fills et porteront mes vêtements à leur place". 
de vache, que lorsque l'enterrement sera terminé, c'est-à-dire lorsque la tombe et le sol de la case mortuaire auront été également enduits de bouse de vache. Partout les femmes enceintes ou réglées s'abstiennent de toucher le cadavre et de s'approcher de la tombe.

L'essentiel des rites de deuil est dirigé par le groupe des bidama. Les bidama étaient autrefois les compagnons de guerre du mort, unis par un même secret de fabrication du poison des flèches. Ils appartiennent à la même unité défensive correspondant, le cas échéant, à une unité résidentielle, concession ou groupe de concessions. Pour certains informateurs, ce lien résiderait plutôt dans une origine paternelle commune. Bien que la règle de résidence soit patrilocale, les agnats ne sont pas automatiquement corésidents; les hasards de l'héritage (un homme peut aller vivre chez son oncle maternel et $y$ demeurer) et les migrations par petits groupes que les Goin pratiquèrent au moins pendant un siècle, ont en effet souvent dispersé les familles. Le groupe des bidama est donc structuré selon un principe résidentiel et/ou agnatique oublié aujourd'hui. Sa seule fonction actuelle est de conduire le rite de l'ambalma pour ses membres.

Les bidama arrivent sur place en même temps que les balafonistes et les fossoyours, tous forgerons ${ }^{7}$. Ils prennent l'arc, le carquois et les flèches du mort, parfois aussi sa houe et son couteau, et les enferment dans une case : ces objets, qui portent le nom générique de hango, représentent le mort jusqu'à l'ambalma. Pendant toute la période de deuil, les veuves donneront chaque jour à boire et à manger au défunt en versant à terre un peu d'eau et de nourriture auprès du hango ( elles effectueront ces gestes dans leur propre case). Les bidama sacrifient un coq pour chacune des veuves afin qu'elles soient protégées des sorciers jusqu'aux funérailles. Leurs cheveux sont rasés puis les bidama leur confectionnent et leur passent autour de la taille une ceinture de fibres de ronier qui ne sera rompue que le

7 En pays goin il y a deux espèces de forgerons: les Tontunamba, d'origine senufo, et les Fonamba, goin. Les premiers sont censés avoir enseigné aux seconds les techniques d'extraction et de fonte du fer. Fossoyeurs et balafonistes peuvent être aussi bien Tontunamba que Fonamba. 
jour de l'ambalma: elle est le signe de la continuation de leur lien conjugal. Les veuves sont tenues à une stricte chasteté durant toute cette période sous peine de tomber gravement malades. Des "soeurs de père" ou des parentes maternelles du mort viennent dormir avec elles jusqu'aux funérailles. On ne les laisse jamais seules la nuit : on les surveille en même temps qu'on les protège des assiduités de leur défunt mari et de leurs éventuels soupirants. Elles ne peuvent quitter le village, ni sortir la nuit sans lumière, et une lampe est allumée en permanence dans leur case pendant la nuit. Hormis ces prescriptions, elles mènent une vie normale et s'acquittent de toutes leurs tâches habituelles.

Les bidama revêtent l'ambalmatiyo du boubou de son père, ils lui donnent ses objets familiers tels que sa gourde, sa canne, son couteau, sa tabatière... Durant toute la période intermédiaire, l'ambalmatiyo endosse la personnalité sociale de son père, il porte ses vêtements, il imite ses attitudes et ses gestes, offrant par exemple du tabac et de la bière de mil aux amis de son père si tel était le comportement de ce dernier; ceux-ci lui répondent comme s'il était le père. On dit qu'"il entre dans le trou de son père", ( $u$ suri $u$ to funguna). Le terme fungo, litt. "trou", par son évocation d'un espace à trois dimensions, suggère que le fils doit occuper la totalité de l'espace social laissé vide par le défunt : non seulement la surface visible de la position sociale du père, mais encore sa dimension cachée dans le monde de l'occulte, celle qui, pour les Goin, donne sa vraie profondeur à un être. On peut faire l'hypothèse que ce trou où le fils doit entrer soit celui de la tombe. La métaphore exprimerait alors qu'en accomplissant les rites de l'ambalma, le fils participe symboliquement au voyage de son père vers l'au-delà, qu'il l'accompagne dans la mort.

Le fils ne peut quitter le village, ni sortir la nuit sans lumière et il est astreint à un certain nombre d'interdits spécifiques à chaque famille et à chaque individu. Il porte en outre des amulettes destinées à le protéger des sorciers. Si le décès a eu lieu pendant la saison des pluies on lui met dans les mains une poignée de terre du champ paternel et on prévient le mort afin que le fils puisse continuer à 
cultiver. Exception faite des objets personnels du défunt que le fils doit porter sur lui, aucune de ses possessions ne peuvent être utilisées avant la fin des funérailles.

\section{L'ambalma}

a) Sortie du hango

Le premier jour de la semaine goin (safiengo), les bidama du mort construisent un paravent de paille qu'ils appuient contre le mur extérieur de la concession, à l'ouest, créant ainsi un "isoloir" derrière lequel l'ambalmatiyo prend place dès le début de la cérémonie $^{\mathbf{a}}$. Il est vêtu d'un costume traditionnel acheté par les maternels du mort : un petit bonnet blanc, une chemise blanche composée de deux pans reliés sous les bras par deux bandes noires, éventuellement une culotte bouffante blanche, les trois pièces étant en coton tissé.

Les bidama sacrifient une poule devant l'entrée de la case mortuaire puis ils entrent à reculons, en enfonçant parfois la porte, après avoir marqué un temps d'arrêt sur le seuil en secouant trois fois leur jambe gauche vers l'arrière et en poussant trois fois le cri de guerre. Lorsqu'il ressortent, cette fois dans le sens habituel de la marche, ils tiennent le hango, c'est-à-dire l'ensemble des objets, armes et outils qui figurent le mort, et ils le posent à terre devant les balafonistes-forgerons qui entonnent le chant des guerriers ${ }^{9}$. Autrefois, on portait le hango au premier carrefour à l'ouest. Aujourd'hui, et bien qu'on désigne toujours ce rite par l'expression "porter le hango au carrefour", la plupart des villages ne s'y risquent plus, de peur qu'un homme qui aurait partagé sexuellement une femme avec le mort ne le voie, ce qui signerait, dit-on, son propre arrêt de mort. Il est en effet interdit à tout homme qui, selon l'éloquente expression goin, "s'est mélangé dans une femme avec le mort", d'entrer dans la concession pendant les cérémonies funéraires, de

\footnotetext{
- A Soubakaniedougou l'isoloir ne sert pas à cacher l'ambalmatipo mais la fabrication de la bière funéraire durant les trois jours précédant le rite.

' Ils peuvent aussi le déposer derrière l'isoloir où se trouve l'ambalmatigo.
} 
boire la bière de mil ou de manger les animaux sacrifiés à cette occasion, et même d'être en contact visuel ou tactile avec quoi que ce soit qui représente ou concerne le défunt.

Le jour de l'enterrement, si le père réel du défunt est décédé, le mort "choisit" l'homme et la femme qui lui tiendront lieu de "père" et de "soeur de père" pendant les funérailles. Autrefois, on pratiquait l'interrogation du cadavre; aujourd'hui cette coutume est abandonnée et on recourt au devin pour connaître les volontés du défunt ${ }^{20}$. Le "père" et la "soeur du père" - qui peuvent être n'importe quelle personne portant le même matronyme que le père réel ${ }^{11}$ et dont le rôle sera plus important à la fin des funérailles - sont responsables du passage de leur "fils" dans l'au-delà.

L'ambalmatiyo a fourni une poule et un coq, ainsi qu'un bouc s'il est déjà marié $\hat{1}^{12}$. Le "père" du mort sacrifie la poule tout près $\mathrm{du}$ hango et dit en substance : "Voici ton fils qui fait ton ambalma. Il va retourner dans la concession avec ton arc. La manière dont tu t'es comporté, il faudra qu'il se comporte pareillement (allusion à la bravoure). Si tout est en ordre, fais que la poule tombe bien, s'il y a un problème, fais le savoir. Laisse la santé aux enfants."

Entre l'enterrement et l'ambalma, de nombreuses consultations divinatoires ont été menées par les maternels et les paternels du défunt : elles ont pour but de faire connaître la cause du décès et de permettre au mort d'avouer tous ses crimes en sorcellerie. Si la poule que le "père" vient de sacrifier près du hango meurt sur le ventre, c'est que le mort est chassé par les ancêtres parce qu'il n'a pas tout avoué ou que ses victimes mettent des obstacles sur son chemin. Des raisons moins essentielles peuvent aussi le pousser à refuser la première poule : tel détail du rituel n'a pas été respecté, il en veut à son fils ou à l'un de ses proches pour tel incident, etc. On essaye de savoir ce qui le contrarie en lui présentant plusieurs

\footnotetext{
10 Dans certaines familles les gens âgés peuvent choisir un "père" de leur vivant.

"La société goin se compose de six matriclans.

${ }^{12} \mathrm{Si}$ l'ambalmatipo est trop jeune pour payer lui-même les animaux sacrificiels, ceuxci lui sont fournis par les maternels du père. A Soubakaniedougou, le fait d'être marié n'entraîne pas l'obligation de fournir un bouc.
} 
poules et en fournissant diverses explications. S'il les refuse toutes, on arrête la cérémonie et on envoie à nouveau les parents chez différents devins pour que le mort puisse achever sa confession. Il arrive aussi, nous a-t-on dit, que l'esprit du mort possède l'un des assistants qui se met brusquement à s'agiter, et à crier les aveux ou les exigences du défunt. Aucun mort ne peut être accueilli par les ancêtres tant qu'il n'a pas avoué la totalité de ses actes de sorcellerie. A chaque aveu, le "père" sacrifie aux ancêtres et il demande que son "fils" soit pardonné. Lorsqu'enfin la poule meurt sur le dos, c'est que la confession est achevée et que les ancêtres lui ont ouvert la porte.

Le "père" du mort peut alors procéder au sacrifice du second animal donné par le fils (un coq). On lui entortille les ailes pour qu'il ne se débatte pas car le sacrifice n'est plus une mantique mais une offrande. Puis, si l'ambalmatiyo est marié, on tue un bouc en l'assommant. Tuer un animal en l'assommant au lieu de l'égorger serait le propre de l'ambalma, car cette "mauvaise" manière de mettre à mort est conforme à la "mauvaise chose" de l'ambalma. Enfin ont lieu les sacrifices des deux poules que les maternels du mort ont offertes pour chaque veuve : un maternel les sacrifie près de l'autel des ancêtres du défunt en leur présentant chaque épouse et en demandant la santé pour elle. Les maternels du mort, ou celui parmi eux qui héritera de la femme, doit fournir un bouc si elle a été adultère ${ }^{13}$.

b) Le repas du hango

Des veuves résidant dans la concession, donc des femmes ayant déjà participé à un ambalma, ont préparé la bière de mil et le repas du hango composê de gâteau de maïs ou de petit mil, de gibier, de poisson et de beignets de pois de terre ou de haricots à l'huile de

13 A Soubakaniedougou, les maternels du mort donnent automatiquement un bouc, partant du principe qu'il est impossible à une ferme d'être fidèle, et on ne présente pas les épouses à l'autel des ancêtres du mari. A Gouera, en revanche, les veuves ne peuvent accomplir l'ambalma de leur mari si cette présentation n'a pas été faite. Autrefois on les présentait lors de leur mariage; actuellement on le fait au moment de l'ambalma, la raison invoquée à cette évolution étant que leur inconduite grandissante leur attirerait trop d'ennuis de la part des ancêtres. 
karité. Un compagnon d'armes du mort (bidayongo, sing, de bidama) donne à l'ambalmatiyo, toujours caché derrière la natte, un simulacre de repas. Il pose trois fois une pincée de ces nourritures alternativement sur la paume, puis sur le dos des mains tendues de l'ambalmatiyo. Trois fois celui-ci retourne ses mains et laisse tomber la nourriture à terre. La quatrième fois, le bidayongo lui met la nourriture dans la bouche et l'ambalmatiyo l'avale. Cette opération sera accomplie quatre fois avec, successivement, une gorgée de bière de mil puis des fragments de pâte de mil, de beignet, de poulet et de bouc, de poisson et de gibier. La nourriture tombée à terre est considérée comme donnée au mort : celui-ci reçoit donc de ses bidama son ultime repas terrestre. L'ambalmatiyo sort alors de sa cachette et, accompagné des bidama, mange le reste du repas du hango et boit la bière de mil dans le gobelet de son père ${ }^{1.4}$. Quant aux veuves, à Gouera, elles ne s'approchent pas du hango, et restent dans la concession $^{15}$. Elles reçoivent le même repas que l'ambalmatiyo et elles le mangent à côté de lui si elles ont été fidèles, à l'intérieur de la concession dans le cas contraire. L'aîné des bidama dépose une pincée de nourriture quatre fois sur le dos puis sur la paume de leurs mains, quatre fois elles la jettent sur un van couvert de bouse de vache, car cette la nourriture ne doit pas toucher terre. La cinquième fois il la dépose dans leur bouche et elles l'avalent. Les miettes seront soigneusement enterrées.

c) Séparation des conjoints et reconduction de l'alliance

Autrefois, un ou deux bidama allaient au marigot confectionner un redoutable objet: le caro ou tosungo, petite brindille de bois entourée de fibre de palmier. Ils revenaient dans la concession et l'un d'eux, le tenant caché dans sa main ou dans un petit panier car les épouses ne devaient pas le voir sous peine de mort, le passait trois fois entre les jambes de chaque veuve en tournant

14 Selon certains informateurs, l'ambalmatiyo ne mangerait aucun repas ce jour-là.

15 A Soubakaniedougou on fait asseoir les veuves au soleil sur le chemin où est déposé le hango jusqu'à ce qu'elles avouent leurs infidélités ; ailleurs elles peuvent accompagner le hango au carrefour. 
autour de la cuisse gauche. Puis on plaçait le caro à côté du hango et il recevait le sang des sacrifices. Les bidama, le futur mari et un maternel de la veuve partaient ensuite l'enterrer dans un endroit secret. Si le nouveau mari, un maternel du mort, était mécontent du comportement de sa femme, il déterrait le caro et en brûlait un morceau, ce qui avait pour effet de rendre celle-ci gravement malade. Aussi était-il fréquent qu'un maternel de l'épouse le dérobe en cachette et le jette ailleurs. Aujourd'hui, dans la plupart des villages, les bidama confectionnent toujours le caro et le placent à côté du hango afin qu'il reçoive le sang des sacrifices, mais ils ne le passent plus entre les jambes des veuves, cette coutume étant considérée comme trop dangereuse pour elles; ils vont ensuite le jeter dans une rivière pour que l'eau l'entraîne. Là où le rite du caro est encore accompli entièrement, il est présenté par les informateurs comme servant à séparer définitivement la veuve de son mari. Lorsque le caro n'est plus passé entre les jambes des veuves, le rite serait destiné à leur assurer santé et fécondité.

\section{Levée du deuil}

A Gouera, où le marigot est toujours à sec en cette saison, ce qu'on appelle le "bain" se déroule dans la cour du mort. Le rite consiste à esquisser trois fois pour les femmes, deux fois pour les hommes, le geste de plonger le pied gauche dans une cuvette d'eau et de l'y plonger réellement la fois suivante. Quand les ambalmatama, veuves et enfants, "reviennent du marigot vers la concession", on rase leurs cheveux qu'on ramasse soigneusement avant qu'ils ne touchent terre. Ils seront portés soit sur la tombe du mort, soit sur un autel d'ancêtre.

Lorsque les veuves ont été purifiées par le bain rituel, les bidama coupent la ceinture de fibres de rônier qu'ils leur avaient nouée autour des reins après le décès. Ils la jettent dans le marigot ou l'enterrent en un endroit secret, ainsi que les deux bouquets de feuilles qu'elles avaient glissés sous cette ceinture au début de l'ambalma pour cacher leur nudité. Les femmes insistent sur l'importance de cette séquence, disant qu'il leur est impossible de se remarier 
tant que les feuilles et la ceinture n'ont pas été jetées. Les bidama leur mettent une nouvelle ceinture végétale que leur prochain mari coupera le soir de leur première nuit de cohabitation. Avant de passer une nuit avec elle, celui-ci devra donner une poule au forgeron, qui la sacrifiera devant la porte de leur case.

Durant toute la durée de l'ambalma, les bidama sont restés à l'extérieur de la concession. La nuit suivante, ils rentrent chez eux sans avoir pénétré une seule fois dans la concession du mort. Autrefois, l'aîné emportait l'arc et les flèches du hango et il les remettait plus tard à l'ambalmatiyo. Ces armes pouvaient retrouver leur usage normal mais jamais elles ne servaient à un autre ambalma ${ }^{16}$. Un bidayongo sacrifie un poulet pour que l'ambalmatiyo puisse rentrer dans la concession de son père. On brûle soigneusement le paravent de paille et tous les débris de l'ambalma; il arrive que les enfants exécutent une ronde autour du bûcher en poussant des cris de guerre.

L'ambalma est terminé. L'ambalmatiyo porte encore pendant un an (ou un mois) le costume blanc qu'il a revêtu pendant le rituel ainsi que la tabatière et la gourde de son père.

\section{Deuil et ambalma d'une femme}

Si les hommes sont représentés à la fin de leur vie par les armes du guerrier et du chasseur, les femmes le sont par les instruments de travail de la porteuse et de la cuisinière. Trois jours seulement avant l'ambalma, on enferme le porte-fagots (kolongo ${ }^{17}$ ) de la défunte dans une case; sa fille lui verse quotidiennement quelques gouttes d'eau et une cuillerée de bouillie de mil. Théoriquement, la case de la défunte est scellée dès l'enterrement afin que son foyer

if. De nos jours, le hango est constitué d'un bâton courbé et de quelques brindilles, reproduction en miniature de l'arc et des flèches; l'un des bidama le démantèle et le jette dans un creux quelconque.

${ }^{12}$ Le kolongo se pose sur la tête. Il est constitué d'un fonds de bois recouvert de peau; ses côtés antérieur et postérieur sont ouverts tandis que ses côtés jatéraux sont fermés par une branche courbée en arceau, de façon à maintenir en place la charge transportée. 
et parfois sa pierre à moudre ne puissent être utilisés pour préparer la nourriture de son mari (en cas de transgression, celui-ci enflerait et mourrait).

Le mari n'est pas tenu à l'abstinence sexuelle et il mène sa vie habituelle. Tout au plus prendra-t-il une lanterne pour sortir la nuit. La ou les filles de la défunte désignées pour l'ambalma évitent de quitter le village et de sortir la nuit sans lumière pendant la période de deuil. Elles ne sont pas tenues de porter les vêtements de leur mère ni d'adopter son comportement. On constate néanmoins que la plupart des filles s'astreignent volontairement à un certain nombre d'interdits qui leur sont davantage inspirés par la piété filiale que commandés par la tradition: porter le pagne de leur mère ou quelques-uns de ses objets personnels, ne pas se laver à l'eau chaude, pratiquer l'abstinence sexuelle...

En ce qui concerne le processus d'ancestralisation, l'ambalma d'une femme a la même nécessité structurelle que celui d'un homme: une femme ne peut devenir ancêtre que si l'une de ses filles, réelle ou classificatoire, accomplit son ambalma. En revanche, du point de vue de la rupture des liens entre vivants et morts et de la libération consécutive des premiers, les rites de séparation sont beaucoup plus simples pour une femme que pour un homme. Si une fille doit être présente à l'ambalma de son père, un fils n'est pas obligé d'assister à celui de sa mère. Un mari n'est formellement tenu d'accomplir que l'ambalma de sa mère. Mais il peut s'y faire représenter par n'importe quel homonyme clanique, tandis qu'une telle délégation est impossible pour une épouse. Par ailleurs, le kolongo - équivalent féminin du hango - est réellement porté au carrefour, c'est-à-dire à un embranchement de sentiers, à l'ouest de la concession. Il n'est pas nécessaire de le cacher car les différents partenaires sexuels de la morte ou les femmes qui auraient partagé un homme avec elle n'encourent aucun danger en le voyant. Aussi l'ambalma d'une femme est-il beaucoup moins dramatisé que celui d'un homme.

La ou les filles du ambalma entrent à reculons dans la case de la morte et ressortent en tenant le kolongo (le porte-fagots), un 
gros panier de riz non décortiqué et quelques objets ayant appartenu à la défunte, tels que van, houe et canne. Elles emportent le tout au carrefour ainsi que la bière de mil spécialement préparée par les veuves. Puis deux maternels du mari (jamais le mari lui-même) entrent à leur tour à reculons dans la case mortuaire, cassent le foyer de la défunte, mettent les morceaux dans un panier et vont les déposer sur le chemin, entre la concession et le carrefour ${ }^{18}$. L'un d'eux sacrifie la poule et la pintade de l'époux près des fragments de foyer et il dit en substance: "Voici ton mari qui a cassé ton foyer. Quand tu étais vivante tu n'as pas refusé de faire à manger un seul jour. Que la paix soit avec toi. Fais que la pintade tombe bien"18. Si la réponse est positive, le rôle du groupe des maris est terminé et il rentre dans la concession. Sinon, on procède à de nouvelles consultations divinatoires. Ensuite, les maternels de la femme sacrifient près du kolongo la poule puis la pintade des filles, le sacrifiant demandant à la morte de laisser les enfants en bonne santé. Les filles retournent alors en courant vers la concession avec le panier de riz et le kolongo sur la tête. Les balafonistes-forgerons qui les attendent à la porte extérieure les bousculent et le riz tombe à terre; ils le ramassent, en gardent une partie pour eux et rendent le reste aux filles, qui font alors parfois le simulacre de le semer. Le van sera déchiqueté et jeté dans un creux. Le kolongo est rapporté dans la case de la morte et la fille qui joue le rôle de l'ambalmatiyo pourra l'utiliser normalement, mais il ne doit jamais resservir pour un autre ambalma.

${ }^{18}$ Le foyer est constitué de deux trous ronds ménagés dans une banquette d'argile qui court le long du mur, à l'intérieur de la case circulaire. Casser le foyer consiste donc à casser l'argile dans la partie de la banquette où se trouvent les deux trous.

${ }^{29}$ a Soubakaniedougou, un mari ne serait tenu à aucun sacrifice pour l'ambalma de sa femme. 
$\mathrm{Au}$ début de cet article, nous avons présenté l'ambalma comme un rituel de deuil poursuivant deux objectifs: l'ancestralisation du défunt et la séparation des vivants et des morts, ce second point nous apparaissant comme la conséquence du premier. Cependant, ne semble répondre pleinement à cette définition que le rite accompli par les enfants pour leur parent de même sexe; celui effectué par lé mari pour sa femme opère de toute évidence une rupture du lien conjugal entre les individus (et non entre les groupes échangistes), mais il n'apparaît pas nécessaire à l'ancestralisation du conjoint défunt. Seuls la participation rituelle et les sacrifices offerts par les enfants sont indispensables à cette transformation du mort. En ce qui concerne l'ambalma accompli pour un homme, il nous a été dit que le défunt se présentait devant les ancêtres avec, à la main, le coq et les poules des enfants et des épouses. Néanmoins, nous faisons l'hypothèse que l'ambalma effectué par une femme pour son mari ne contribue pas non plus à l'ancestralisation du défunt mais seulement à la séparation des conjoints.

"On ne fait l'ambalma qu'une seule fois dans sa vie, parce que personne n'est assez fort pour le faire deux fois sans mourir" disent les Goin. Or nous avons vu qu'une femme devait effectuer l'ambalma de sa mère et de son premier mari et qu'un homme accomplissait le rite pour son père et pour son (ou ses épouses) non héritées, s'il le souhaitait. La formule goin, qui ne précise pas quel est ce destinataire unique du rite, semble exclure les ambalma effectués pour les conjoints et ne prendre en compte que l'ambalma qu'effectue un fils pour son père, une fille pour sa mère. Un ambalma au sens plein du mot serait donc un rite qui "enlève la mauvaise chose du mort", qui le purifie des souillures accumulées pendant sa vie terrestre et qui, de ce fait, lui permet de devenir ancêtre. Ce serait ensuite, mais par simple voie de conséquence, un rite de séparation entre morts et vivants. L'ambalma accompli par un conjoint, dans la mesure où il ne contribue pas à l'ancestralisation de celui-ci mais seulement à la rupture des liens avec lui, ne mériterait pas pleinement le nom d'ambalma et ne serait appelé ainsi que par extension du terme. 


\section{PARENTS EN DEUIL ET PARENTS HORS DEUIL}

Les maternels, qui héritent les épouses et les biens mobiliers du défunt, assument la plus grande partie des dépenses des cérémonies funéraires (animaux sacrificiels et surtout repas et boisson pour les invités). Les aînés discutent en conseil de famille des politiques financières à adopter au sein du matrilignage et décident des dates auxquelles ils pourront faire face à tout ou partie des dépenses afférentes à ces cérémonies. Malgré l'importance de leur rôle et l'étroitesse de leur lien de parenté avec le mort, on constate que les maternels ne sont soumis à aucun comportement ou marque de deuil. Seuls les conjoints et les enfants, c'est-à-dire les parents issus de l'alliance, adoptent les comportements et accomplissent les rites de deuil, seuls ils ont à couper rituellement les liens qui les unissent au mort. Nous nous proposons d'examiner maintenant les raisons de cette distribution des rôles entre parents en deuil et parents hors deuil.

\section{Le groupe de filiation}

La filiation dominante est matrilinéaire. L'unité sociale de base, le matrilignage, est un groupe en corps (corporate group) exogame, possesseur collectif du numéraire et des biens qui peuvent être acquis avec de l'argent, au premier rang desquels se placent les épouses et les captifs. Ce groupe comprend évidemment les ancêtres: l'aîné des vivants leur rend un culte au nom et au bénéfice de tous les membres du groupe.

Dans la conception et la gestation, la mère est considérée comme responsable de la moitié de la substance physique de l'enfant; à la naissance, celui-ci est intégré automatiquement à son matrilignage puisque "c'est le même sang" ou "le même lait". Pour employer les termes de Leach $^{20}$, chacun est avec ses maternels dans un rapport d'incorporation permanente. 
Paternité et alliance

L'autre moitié de la substance physique de l'enfant lui est donnée par son géniteur, mais cette hérédité biologique est dépourvue de toute conséquence psychique et spirituelle directe. D'autre part, la paternité n'est pas conférée par l'engendrement mais par l'alliance. Les Goin connaissaient (et connaissent parfois encore) une double union: la première, conclue entre jeunes, s'achevait après quelques années de cohabitation par une cérémonie appelée kubenao (c"est frais"), au cours de laquelle la jeune femme était excisée. Son amant payait les frais du rituel et de la fête de trois jours qui s'ensuivait. En outre, il versajt à l'oncle maternel de la jeune femme une compensation équivalente à quinze mille francs CFA actuels et fournissait, pendant toute la période de cohabitation, des prestations en travail aux parents de son amie. Après ce rite, la jeune femme demeurait encore chez lui quelques semaines, quelques mois au plus, puis avait lieu une seconde cérémonie, kukurao ("c'est sec"), à l'issue de laquelle elle était mariée avec son fiancé officiel, obligatoirement distinct de l'amant. Dès lors, elle ne devait plus entretenir de relations avec son amant. Le mari, à qui la fille était obligatoirement promise depuis sa naissance, payait les frais du kukurao, versait les mêmes prestations que l'amant aux oncles maternels de sa femme, donnait à celle-ci les mêmes cadeaux et fournissait également aux parents de sa fiancée des prestations en travail (légèrement inférieures à celles de l'amant). Mais, alors que les prestations de l'amant ne lui conféraient aucun droit sur la femme ou sur les enfants qu'il pouvait engendrer, celles du mari lui donnaient des droits exclusifs sur la femme et surtout des droits de paternité permanents sur la progéniture de celle-ci, quels que soient le géniteur et l'époque de la conception.

Actuellement l'ami tend à devenir le mari, mais les droits conjugaux et paternels ne sont acquis que lorsque le prétendant a accompli successivement les deux cérémonies et achevé les deux séries de prestations. L'important pour l'accès à l'alliance et à la paternité n'est pas que la séquence rituelle comprenant kubenao et kukurao soit accomplie par un ou deux hommes mais qu'elle soit 
accomplie entièrement et que la double série de prestations soit effectuée.

Les enfants sont placés sous la dépendance spirituelle du mari de leur mère que nous appellerons leur père social. Il est responsable des deux grands passages rituels de leur vie : le mariage et les funérailles. Il peut, seul avec la mère, les tuer d'une malédiction ou livrer leur double ancestral au sorcier. Les fils qui ont fait l'ambalma, ainsi que l'aîné, succèdent à leur père social dans l'ensemble de ses positions magico-religieuses. Tous ses enfants sont sous son "influence métaphysique" ou "influence mystique" (Leach, op. cit.).

Les enfants, lorsqu'ils étaient issus de l'amant, connaissaient l'homme qui les avait engendrés mais ils n'entretenaient aucune relation avec lui puisqu'aucun lien social ne les unissait. Dans la mesure où, autrefois, l'amant n'épousait jamais la mère, il n'était jamais le père social de ces enfants et ceux-ci ne faisaient en aucun cas son ambalma. Une seule circonstance pouvait les mettre ponctuellement en contact : chacun possède un double ancestral, le jufwengo, constituant spirituel et psychique essentiel de la personne, maître de son destin de surcroît. Or le jufwengo peut provenir d'un ancêtre, d'un ami ou d'une amie de l'un des deux géniteurs. L'identité du jufwengo doit être tenue secrète : lorsque le devin révélait que le jufwengo d'un enfant d'amant provenait de son géniteur, la mère n'en dévoilait jamais l'identité à son mari, le père social de l'enfant. Si le jufwengo réclamait un sacrifice, la mère partait avec l'enfant chez son ancien amant, seul habilité à se charger des modalités rituelles requises. Le père social ne devait en aucun cas s'en occuper.

Dans le tableau I, qui résume l'ensemble des déterminants de la personne et du destin individuel, on voit que la paternité biologique et ses conséquences sur l'hérédité ne sont pas prises en compte. Elles sont même déniées lorsque le géniteur est l'amant. Les Goin disent : "La paternité, c'est 1'alliance". Il faut ajouter que la formule est réversible : l'alliance est la paternité, et ceci, que le mariage ait été fécond ou non, comme il apparaît clairement lors de l'ambalma: un homme marié sans descendance aura toujours un "fils" pour 
accomplir son ambalma tandis qu'un homme non marié n'aura pas d'ambalma, quel que soit le nombre des enfants qu'il aura engendrés.

\begin{tabular}{|c|c|c|}
\hline Origine & Eléments de la personne & Conséquences sociales \\
\hline mère & $\begin{array}{l}\text { moitié de la substance } \\
\quad \text { corporelle } \\
\text { légère influence mysti- } \\
\text { que sur ses filles }\end{array}$ & $\begin{array}{l}\text { intègre l'enfant à } \\
\text { son matrilignage }\end{array}$ \\
\hline géniteur & $\begin{array}{l}\text { moitié de la substance } \\
\text { corporelle }\end{array}$ & nulles \\
\hline $\begin{array}{l}\text { père social } \\
\text { (qu'il soit } \\
\text { ou non le } \\
\text { géniteur) }\end{array}$ & $\begin{array}{l}\text { grande influence } \\
\text { mystique surtout } \\
\text { sur ses fils }\end{array}$ & $\begin{array}{l}\text { les fils lui succède- } \\
\text { ront dans toutes ses } \\
\text { fonctions magico-reli- } \\
\text { gieuses }\end{array}$ \\
\hline jufwengo & $\begin{array}{l}\text { personnalité, destin, } \\
\text { mesure de la force } \\
\text { dans le monde occulte }\end{array}$ & $\begin{array}{l}\text { globalement responsable } \\
\text { de la personnalité, } \\
\text { du comportement, du } \\
\text { destin de l'individu }\end{array}$ \\
\hline
\end{tabular}

Tableau 1

La paternité n'a d'existence que si elle est affirmée par les rites: le premier est le mariage, le second est l'ambalma. Avant de montrer que ces deux rites font système, il faut dire un mot de l'organisation de la descendance.

\section{La descendance}

Pour une femme descendance et filiation se confondent tandis que pour un homme elles sont évidemment disjointes. Il n'existe ni patrilignage ni culte ancestral agnatique, mais seulement une relation individuelle père-fils et un culte rendu par le fils à son père défunt. Encore ne peut-il ni l'instaurer ni l'accomplir lui-même, il doit demander la médiation d'un utérin du père, tant il est vrai qu'un ancêtre stricto sensu ne peut être que maternel. Au cours de l'ambalma, un fils contribue à l'ancestralisation de son père pour le 
bénéfice du groupe de filiation de ce dernier. Une pierre, représentant le père ancestralisé, est automatiquement placée, à l'issue des funérailles, dans l'autel lignager des maternels par leur aîné. Si le père veut être honoré à titre individuel par son fils, il l'en avertira par la médiation du devin et le fils demandera au neveu utérin du père de lui fabriquer l'autel (un caillou fixé au mur extérieur d'une case) à l'écart de l'autel des ancêtres maternels (des rangées de pierres fixées au mur de l'autre côté de la porte ou sur une autre case). Une fois l'autel posé, les modalités du culte sont moins rigides: pour les uns, le fils est libre de s'adresser directement à son père et de sacrifier lui-même; pour les autres, il doit demander à un maternel du père - ou à n'importe qui ayant le même matronymede tenir le couteau et de transmettre ses paroles. En revanche, dans d'autres domaines du religieux (celui des cultes agraires, ou celui des magies et des "fétiches" donnés à titre individuel par les génies ou par Dieu, et transmis de père en fils), le fils sera officiant du culte et invoquera non l'entité propitiée mais son père, médiateur obligé entre lui et la puissance concernée.

La relation agnatique est, disions-nous, une relation individuelle entre deux personnes, le père et le fils. Mais, dans la mesure où le père a lui-même un père, il s'établit une relation entre trois personnes: le grand-père paternel, le père et le fils. L'ambalma est une des rares circonstances de la vie goin où la relation entre ces trois personnes s'actualise simultanément.

\section{Ambalma et mariage}

Le mariage fonde la paternité. La naissance d'un enfant, qui ne représente rien en soi du point de vue de la descendance masculine, est très peu ritualisée. Lorsqu'un homme adulte et marié meurt, même s'il est encore trop jeune pour obtenir des funérailles complètes, même s'il n'a pas engendré d'enfants, il a droit à un ambalma, donc à un "fils" qui accomplira le rite pour lui.

L'ambalma nécessite également un "père" et une "soeur de père". Nous avons dit plus haut comment le mort choisissait ces "parents", qui peuvent être toute personne portant le même matronyme que le 
père social : on dépasse ici l'équivalence structurelle habituelle entre membres du matrilignage ainsi que la parenté classificatoire ordinaire pour atteindre une équivalence structurelle s'étendant aux membres du matriclan.

Pendant le temps du rite, la fonction paternelle est dédoublée: elle a pour support, d'une part le père-ancêtre du mort, d'autre part son substitut vivant que nous désignerons par : "père", gardant la graphie: père pour l'ensemble de la fonction paternelle. L'ambalma met en relation un mort, son père, sous sa double forme vivante et ancestralisée, et son fils, réel ou classificatoire. Le rite actualise le moment où le mort se présente devant la communauté des ancêtres, son père-ancêtre jouant, dit-on, le rôle de médiateur. Ceux-ci refuseraient de l'accueillir sans le rituel accompli sur terre par son "père" et par son fils et, plus précisément, sans l'offrande des animaux sacrificiels du fils. Rappelons que le défunt ne peut être ancestralisé qu'à condition d'avoir avoué devant les vivants la totalité des actes de sorcellerie commis pendant sa vie terrestre. Tant qu'il ne l'a pas fait les ancêtres lui refusent l'accès à leur "village"; il le signale aux vivants en rejetant les poules du fils. Ceux-ci lui donnent alors la parole par l'intermédiaire du devin afin qu'il puisse achever sa confession. Puis le "père" recommence à sacrifier des poules données par le fils jusqu'à ce qu'elles tombent du bon côté. On dit que le mort se présente devant les ancêtres avec, à la main, le coq et les poules offerts par ses enfants et ses épouses. Sans ces animaux, la honte l'empêcherait de s'approcher des autres morts.

Ainsi l'ambalma met en scène une relation de dépendance entre le mort et son père, le mort et son fils. Le père est considéré comme un médiateur : en tant que père-ancêtre il est le médiateur entre son fils mort et la communauté des ancêtres; en tant que "père", acteur essentiel du rite de l'ambalma, il l'est entre le mort et la communauté des vivants, dont le fils est le représentant principal. Dans la pensée goin, cette médiation accomplie par le père entre son enfant et la totalité visible et invisible du monde est inhérente à l'essence même de la relation paternelle. 


\begin{tabular}{|c|c|c|}
\hline & $\begin{array}{r}\text { RITES MATRIMONIAUX } \\
\text { STEREITE }\end{array}$ & $\begin{array}{l}\text { RITES FUNERAIRES } \\
\text { ET MORT }\end{array}$ \\
\hline $\begin{array}{l}\text { kubenao } \\
\text { (c'est } \\
\text { frais }\end{array}$ & $\begin{array}{l}\text { Excision, "première danse" } \\
\text { mort symbolique } \\
\text { Passage initiatique de } \\
\text { l'adolescent à l'âge } \\
\text { adulte } \\
\text { - Frais payés par l'amant } \\
\text {. Sacrifices présidés par le } \\
\text { père de la fille } \\
\text { Convalescence: début de la } \\
\text { période de stérilité tempo } \\
\text { raire }\end{array}$ & $\begin{array}{l}\text { Mort physique, enterre- } \\
\text { ment, traitement du corps } \\
\text { séparé des constituants } \\
\text { spirituels } \\
\text { - Frais payés par les } \\
\text { maternels } \\
\text {. Sacrifices présidés par le } \\
\text { père } \\
\text { Début de la période de } \\
\text { deuil pour les alliés et } \\
\text { les enfants } \\
\text { Début de la période } \\
\text { d'errance pour le mort }\end{array}$ \\
\hline $\begin{array}{l}\text { Période } \\
\text { inter- } \\
\text { médiai- } \\
\text { re }\end{array}$ & $\begin{array}{l}\text { Période dangeureuse pour la } \\
\text { fille } \\
\text { Danger de contagion de ses } \\
\text { émanations stérilisantes } \\
\text { Chasteté, parenthèse dans } \\
\text { sa fécondité, donc dans la } \\
\text { possibilité de transmigra- } \\
\text { tion dans son utérus d'es- } \\
\text { prits ancestraux, jufwengo }\end{array}$ & $\begin{array}{l}\text { Période dangeureuse pour } \\
\text { les deuilleurs } \\
\text {. Nombreux interdits dont } \\
\text { chasteté des épouses } \\
\text {. Pour le mort, attente } \\
\text { entre deux statuts }\end{array}$ \\
\hline $\begin{array}{l}\text { kukurao } \\
\text { (c'est } \\
\text { sec) }\end{array}$ & $\begin{array}{l}\text { "Deuxième danse" : mariage } \\
\text { pour la femme, retour des } \\
\text { possibilités de fécondité, } \\
\text { Pour l'homme, accès à la } \\
\text { paternité } \\
\text { Pour les ancêtres, } \\
\text { réouverture du cycle de } \\
\text { transmigration } \\
\text {. Frais payés par le mari } \\
\text {. Sacrifices présidés par le } \\
\text { père de la mariée }\end{array}$ & 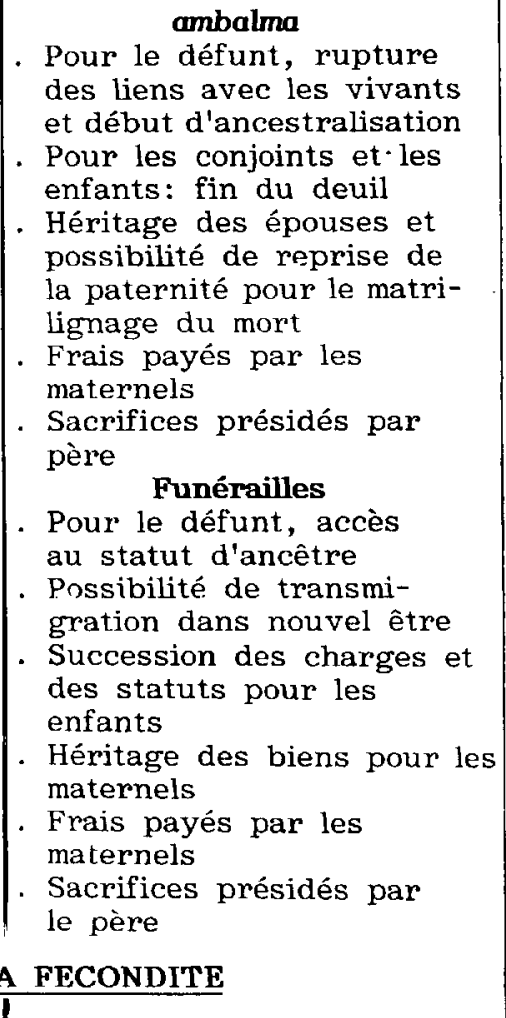 \\
\hline
\end{tabular}


Le "père" du défunt actualise rituellement sa relation de pateruité pour la seconde fois de sa vie, la première étant son mariage; le fils actualise pour la première fois sa position de fils; le mort "actualise", si l'on peut dire, sa relation à son père et à son fils. La collaboration des générations alternées nécessaires à l'ancestralisation de leur terme médian rappelle le principe de leur équivalence structurelle.

Le rite de mariage a fondé la paternité du père, le rite ambalma oblige le fils à assumer sa position de fils et c'est en quoi les deux rituels sont symétriques et complémentaires. En effet, si l'on compare les trois temps des rites funéraires et des rites matrimoniaux, on constate qu'ils se correspondent terme à terme. L'enterrement se nomme kubenao, "c'est frais"; il est suivi d'une période intermédiaire, la période de deuil, qui s'achève par l'ambalma, premier acte des funérailles, lesquelles se nomment kukurao, "c'est sec". Pendant cette période intermédiaire, le mort n'a pas quitté complètement le monde des vivants, il n'est pas encore entré dans celui des ancêtres, il ne peut pas recommencer son cycle de vie, il est dangereux pour ses proches. De même la jeune femme, entre l'excision, kubenao, et le mariage, kukurao, est hors du cycle de fécondité; non seulement elle est temporairement stérile mais encore sa stérilité est contagieuse et peut, par l'intermédiaire du sang impur qui coule de sa blessure, contaminer les champs et les hommes; elle-même est comme les deuilleurs en grand danger d'être ensorcelée. En effet les jeunes excisées doivent éviter tout contact avec les "choses" de la mort: rencontrer une procession funéraire, boire par mégarde la bière des funérailles ou toucher un objet ayant été en contact avec un mort entraînerait, dit-on, la mort des jeunes filles.

$\mathrm{Si}$ on replace en vis-à-vis (cf. tableau ci-contre) les rites matrimoniaux et les rites funéraires, on constate qu'ils ont la même structure formelle. 
Deuil, filiation et descendance d'un homme

On voit maintenant pourquoi seuls les conjoints et les descendants prennent le deuil : du point de vue des individus, la mort rompt le lien d'alliance et sa conséquence, la paternité, mais du point de vue des groupes de filiation auxquels appartiennent ces individus, les liens perdurent et sont transférés du membre décédé à un membre vivant. Instaurés par un rite, le mariage, les liens d'alliance et de paternité doivent être reconduits par un autre rite: l'ambalma.

Le fils est non seulement le produit de l'alliance du père mais aussi un allié par rapport au père : d'une part parce qu'ils appartiennent à deux lignages alliés, d'autre part parce qu'ils sont partenaires actifs et permanents de l'alliance. Le père doit offrir à son fils sa première épouse tandis que le fils lui rend la deuxième fille issue de ce mariage. L'idéal pour les Goins est que ce cycle d'échange, hautement valorisé, se reproduise aussi souvent que possible dans leur vie.

Le fils n'est pas qu'un allié du père, il est aussi son descendant, c'est-à-dire celui que le père a socialement engendré et qui reprendra l'ensemble des positions sociales paternelles non liées à la filiation. Le deuil et l'ambalma célèbrent la capacité d'engendrement symbolique du père. Lors de l'ambalma, le fils, entouré des compagnons d'armes du père, collabore à un double changement statutaire : il aide son père à acquérir un statut chez les ancêtres, et donc à quitter celui qu'il occupait de son vivant; il est lui-même autorisé à prendre graduellement possession de la place ainsi libérée.

En revanche, au sein du groupe des maternels, les comportements de deuil et les rites de séparation n'ont pas à être marqués pour deux raisons :

- Les maternels sont un groupe en corps comprenant les vivants et les morts. La même substance physique et mystique, la même volition, les mêmes intérêts animent les membres vivants et morts du matrilignage. Dans ces conditions, la transformation rituelle du cadavre en 
ancêtre ne peut être prise en charge par les alter ego vivants du mort. Cette responsabilité revient aux alliés : son père et son fils ${ }^{21}$. Mais, une fois le processus mené à terme par la ligne agnatique du défunt, les maternels reprennent possession de leur mort et le fixent, sous la forme d'une pierre, dans l'autel des ancêtres du matrilignage.

- L'appartenance au groupe des maternels n'est pas fonction de l'alliance mais de la mise au monde par la mère. Si la relation mèreenfant fait l'objet d'un traitement symbolique au même titre que la relation père-enfant, il n'en demeure pas moins que celle-là se fonde sur une donnée d'évidence dont ne dispose pas la paternité: l'accouchement de la mère. Il s'ensuit que la paternité plus que la maternité nécessite une expression symbolique et rituelle par le mariage et les funérailles.

Deuil d'une femme : filiation et descendance

Pour les femmes la relation mère-fille est également célébrée, bien que plus modestement, au cours des cérémonies funéraires. Cette relation mère-fille recouvre deux liens différents qu'il convient de distinguer : 1'un de descendance et l'autre de filiation. Lorsqu'une fille accomplit l'ambalma de sa mère elle le fait non en tant que membre du même groupe de filiation mais en tant que descendante. Dans la plupart des circonstances de la vie, la filiation masque la descendance car la filiation est le cadre majeur des activités socioéconomiques. Cependant les rites et comportements de deuil sont pour les femmes, comme pour les hommes, l'affaire exclusive de la descendance. C'est le moment privilégié où ce lien ascendant-descendant est célébré, indépendamment de sa position dans ou hors de la filiation dominante. Ainsi l'opposition introduite par Meyer Fortes

21 De même, dans Death, property and the ancestors, a study of the mortuary customs of the LoDaqaa of West Africa (Tavistock Publications, London, 1962), J. Goody insiste sur le fait que la plupart des rituels funéraires ne sont pas accomplis par les parents les plus proches du mort mais par ce qu'il appelle des partenaires d'affliction au sein de réseaux d'échanges de services d'affliction. 
entre filiation dominante/descendance complémentaire n'épuise pas le champ de la parenté goin: elle escamote la relation de descendance lorsque celle-ci se situe à l'intérieur de la filiation dominante.

\section{Conclusion}

Les cérémonies de deuil, et plus largement les cérémonies funéraires, demeurent, malgré l'acculturation rapide de la société goin, un phénomène d'une très grande richesse, où se révèlent tous les aspects de la réalité sociale, y compris ceux que l'on ne peut pas observer, ou observer aussi clairement, en d'autres circonstances: ainsi l'opposition structurelle entre descendance et filiation pour une femme; le couplage deux à deux des six matriclans goin; la relation à plaisanterie des Goin et des Lobi qui ne se manifeste plus guère qu'aux enterrements où les Lobi, s'il s'en trouve dans le voisinage, viennent jouer un rôle de bouffon en caricaturant les pleurs des proches; la relation entre bidama totalement obsolète et jamais actualisée en dehors de l'ambalma; le rôle essentiel de ces derniers dans le contrôle de la fidélité des épouses et ce qu'il révèle des conceptions goin à ce sujet; les chants des balafonistes-forgerons qui disent en cette occasion ce qu'on ne proclame pas dans la vie ordinaire, par exemple que le défunt est descendant de captif. D'autre part la totalité des relations sociales du mort est mise à contribution pendant les cérémonies funéraires. On s'aperçoit alors qu'aucune relation, si apparemment privée, si libre, si minime soitelle, n'échappe aux codes sociaux : ainsi les amis du mort qui avaient conclu un pacte d'amitié avec lui vont se livrer auprès de ses descendants à une offre de reprise de contrat; les femmes pour qui le mort fut un intermédiaire dans leur mariage apporteront le savon pour sa toilette funéraire...

La société goin s'est beaucoup transformée pendant les trente dernières années et les vieux ne cessent de déplorer la décadence des cérémonies funéraires. Cependant celles-ci demeurent un phénomène social total apte à refléter aussi bien un stade ancien de la 
société que ses innovations les plus récentes. Ainsi, lorsqu'un villageois musulman meurt, comme on ne peut pas réunir dans un même ensemble rituel des réalités aussi hétérogènes que les rites musulmans et l'ambalma, on a recours à deux cérémonies successives, espacées de quelques heures; jusqu'à présent on ne s'est pas encore résolu dans les villages à renoncer aux rites traditionnels.

Michèle Dacher

E.H.E.S.S. 